\title{
Identification of novel biomarker candidates by proteomic analysis of cerebrospinal fluid from patients with moyamoya disease using SELDI- TOF-MS
}

Yoshio Araki ${ }^{1,2^{*}+}$, Kazuhiro Yoshikawa ${ }^{2,3 \dagger}$, Sho Okamoto $^{1 \dagger}$, Masaki Sumitomo ${ }^{1 \dagger}$, Mikio Maruwaka ${ }^{1 \dagger}$, Toshihiko Wakabayashi ${ }^{1+}$

\begin{abstract}
Background: Moyamoya disease (MMD) is an uncommon cerebrovascular condition with unknown etiology characterized by slowly progressive stenosis or occlusion of the bilateral internal carotid arteries associated with an abnormal vascular network. MMD is a major cause of stroke, specifically in the younger population. Diagnosis is based on only radiological features as no other clinical data are available. The purpose of this study was to identify novel biomarker candidate proteins differentially expressed in the cerebrospinal fluid (CSF) of patients with MMD using proteomic analysis.

Methods: For detection of biomarkers, CSF samples were obtained from 20 patients with MMD and 12 control patients. Mass spectral data were generated by surface-enhanced laser desorption/ionization time-of-flight mass spectrometry (SELDI-TOF-MS) with an anion exchange chip in three different buffer conditions. After expression difference mapping was undertaken using the obtained protein profiles, a comparative analysis was performed.

Results: A statistically significant number of proteins (34) were recognized as single biomarker candidate proteins which were differentially detected in the CSF of patients with MMD, compared to the control patients $(p<0.05)$. All peak intensity profiles of the biomarker candidates underwent classification and regression tree (CART) analysis to produce prediction models. Two important biomarkers could successfully classify the patients with MMD and control patients.

Conclusions: In this study, several novel biomarker candidate proteins differentially expressed in the CSF of patients with MMD were identified by a recently developed proteomic approach. This is a pilot study of CSF proteomics for MMD using SELDI technology. These biomarker candidates have the potential to shed light on the underlying pathogenesis of MMD.
\end{abstract}

\section{Background}

Moyamoya disease (MMD) is characterized by progressive stenosis or occlusion of the bilateral internal carotid arteries associated with compensatory abnormal vascular network, so called moyamoya vessels [1]. A Japanese survey of 2075 patients with MMD found an annual incidence of 0.35 , annual prevalence of 3.16 per 100,000 and a

\footnotetext{
* Correspondence: yusei.a.kanna@gmail.com

+ Contributed equally

'Department of Neurosurgery, Nagoya University Graduate School of Medicine, 65 Tsurumai-cho, Showa-ku, Nagoya, Aichi 466-8550, Japan Full list of author information is available at the end of the article
}

tendency of occurrence in the younger generation [2]. According to a world distribution analysis, a relatively large number of patients with MMD are present in East Asia, but rarely in Europe and the Americas [3]. Such regional and racial differences in susceptibility and familial occurrence in $10 \%$ of MMD cases [4] suggest that a genetic predisposition may be associated with the etiology and pathogenesis of this disease. Both 3p24-26 [5] and $8 \mathrm{q} 23$ [6] in genome-wide analyses, in addition to both 6q25 (D6S441) [7] and 17q25 [8] in chromosomal level analyses, have been identified in familial MMD and were 
recognized as possible sources of MMD. Pathologically, stenosis or occlusion of internal carotid arteries has been attributed to eccentric fibrocellular thickening of the intima following proliferation and necrosis of smooth muscle cells, which are associated with the thinning of the media $[9,10]$. These processes are reported to be regulated by the expression of several growth factors related to angiogenesis: transforming growth factor- $\beta$ [11], basic fibroblast growth factor [12], hepatocyte growth factor [13], hypoxia inducible factor-1 [14] and vascular endothelial growth factor [15]. An increasing number of reports have been focusing on not only angiogenesis related to growth factors but vasculogenesis. Vasculogenesis is considered the pathway for adult neovascularization, which induces the formation of new blood vessels from circulating bone marrow-derived endothelial progenitor cells rather than from local endothelial cells regulated by growth factors $[16,17]$. It has been hypothesized that aberrant vasculogenesis contributes to vascular abnormalities including MMD [18]. Despite the establishment of approaches for clarifying the disease mechanisms of MMD, the direct underlying pathogenesis remains unclear. One approach utilizing proteomics has revealed disease-associated proteins as novel biomarkers and characterized their function in pathogenesis and development of the disease $[19,20]$. Among the many different types of approaches for CSF investigation [21], SELDI-TOF-MS technology [22] allows for high-throughput analysis of samples with diverse functionalization on surfaces (ProteinChip, Bio-Rad Laboratories, Hercules, CA, USA) and has been successfully used to identify protein profiles of central nervous system disorders [23,24]. The objective of this study was to apply SELDI-TOF-MS technology to identify candidate proteins in the CSF for use as biomarkers of MMD.

\section{Methods}

\section{Patient Population}

For proteomic analysis, CSF samples were prospectively collected from a consecutive sequence of 20 patients with MMD (11 male and 9 female; mean age, 21 years; range 154 years) admitted to Nagoya University Hospital, Nagoya, Japan, between February 2008 and December 2009. Diagnosis of MMD was determined by cerebral angiography or magnetic resonance imaging/angiography according to the guidelines set by the Research Committee on Moyamoya Disease (Spontaneous Occlusion of Circle of Willis) of the Ministry of Health and Welfare of Japan [25]. Type of onset of MMD includes 12 transient ischemic attacks, four cerebral infarctions, one hemorrhage and two asymptomatic cases. According to the guidelines for the diagnosis of MMD, "definite MMD" cannot have originated from any other underlying disease; the presence of an associated disease is classified as "quasi-MMD". On the other hand, for proteomic analysis of the CSF used in this study, results are thought to be unsusceptible to the underlying disease. That is, both classifications of the disease (definite or quasi) have similar clinical characteristics and treatments. Therefore, two of the patients in this study presenting with Down syndrome were categorized as MMD (Table 1). As controls, 12 patients were recruited from among those admitted to Nagoya University Hospital for

Table 1 Clinical characteristics of each patient group in this study

\begin{tabular}{|c|c|c|c|c|}
\hline & & No. & (\%) & \\
\hline & & MMD & Controls & $P$ value \\
\hline & Variables & $\mathrm{n}=\mathbf{2 0}$ & $n=12$ & \\
\hline Age & Mean $\pm S D$, years & $20.5 \pm 17.4$ & $61.2 \pm 9.1$ & $<0.01$ \\
\hline Sex, n (\%) & & & & \\
\hline & Male & $11(55.0)$ & $3(25.0)$ & 0.1 \\
\hline & Female & $9(45.0)$ & $9(75.0)$ & \\
\hline Type of onset, n (\%) & & & & NA \\
\hline & $\mathrm{TIA}$ & $12(60.0)$ & & \\
\hline & Infarction & $4(20.0)$ & $2(16.7)$ & \\
\hline & Hemorrhage & $1(5.0)$ & & \\
\hline & Asymptomatic & $2(10.0)$ & $7(58.3)$ & \\
\hline & Others & $1(5.0)$ & $3(25.0)$ & \\
\hline Associated disease, n (\%) & & & & NA \\
\hline & None & $18(90.0)$ & & \\
\hline & Down syndrome & $2(10.0)$ & & \\
\hline & Cerebral aneurysm & & $9(75.0)$ & \\
\hline & Others & & $3(25.0)$ & \\
\hline Suzuki's angiographycal stage (1-6) & Mean $\pm S D$ & $3.05 \pm 0.9$ & & NA \\
\hline
\end{tabular}

$\mathrm{TIA}$, transient ischemic attack; MMD, moyamoya disease; SD, standard deviation. 
surgical treatment of other clinical conditions, including nine cases of cerebral aneurysm. The control patients were matched according to gender; however, their overall age was older than that of patients with MMD, as patients with MMD tend to be younger than those with cerebral aneurysm (75\% of control patients), which mainly affects middle-aged individuals.

\section{Sample Collection}

All CSF samples were collected after obtaining informed written consent from the patients following approval from the Nagoya University School of Medicine Ethical Review Board. CSF was obtained for all subjects in the same manner during the first phase of the operative procedure after the arachnoid membrane was dissected. Blood contamination was avoided as much as possible by carefully performing hemostasis. CSF sampling was performed at least 4 weeks after the stroke event associated with MMD or other pathogenesis. Within $2 \mathrm{~h}$ of collection, the CSF sample was stored in $1.5-\mathrm{ml}$ cryotubes, centrifuged at $3000 \mathrm{rpm}$ for $10 \mathrm{~min}$ at $4^{\circ} \mathrm{C}$, and the supernatants were frozen and stored in approximately $1-\mathrm{ml}$ aliquots at $-80^{\circ} \mathrm{C}$ until analysis.

\section{Sample preparation and analysis CSF preparation}

The CSF samples in each cryo-tube were thawed in water at room temperature, then kept on ice and well agitated before subjected to ProteinChip array. The samples were diluted by each binding/washing buffer $(0.2 \mathrm{M}$ citrate-phosphate, $\mathrm{pH} 5$ or 7 , or $0.2 \mathrm{M}$ Tris- $\mathrm{HCl}, \mathrm{pH} 9$ ) at a final ratio of $1 / 5$.

\section{ProteinChip array pretreatment}

Q10 (strong anion exchanger) ProteinChip array (BioRad Laboratories) was used for protein profile analysis. The chips were equilibrated prior to the CSF sample addition with binding/washing buffer for $2 \times 5$ min.

\section{ProteinChip array preparation}

Diluted CSF $(20 \mu \mathrm{l})$ was applied on a spot in the chip and incubated for $40 \mathrm{~min}$ in a humid box at room temperature. After allowing the sample to bind, the remaining sample was removed from all spots by immediately washing the array with the corresponding binding/washing buffer $3 \times 5 \mathrm{~min}$. The arrays were rinsed with $150 \mu \mathrm{l}$ distilled water twice and allowed to air-dry for $15 \mathrm{~min}$. Then, $1 \mu \mathrm{l}$ saturated energy absorbing molecule (EAM) solution (sinapinic acid in $50 \%$ acetonitrile and $0.5 \%$ trifluoroacetic acid) was applied to each spot and again allowed to air-dry before analysis by the ProteinChip reader.

\section{SELDI-TOF-MS and data acquisition} Mass spectral processing

The protein mass spectral data was generated with ProteinChip System 4000 SELDI-TOF mass spectrometer
(Enterprise version; Bio-Rad Laboratories) using automated data collection protocol with Ciphergen Express version 3.0.6 software interface (Bio-Rad Laboratories). The optimal laser intensity range for spectra generation was predetermined to be 2800 to $3500 \mathrm{~nJ}$ by manual laser shots. For determining the maximum protein peak yield and spectral reproducibility, data were collected between 0 and $100 \mathrm{kDa}$ with the ion focus mass set to $7000 \mathrm{Da}$ and the matrix attenuation to $1000 \mathrm{Da}$. After two initial warming shots, 53 points equally distributed over the chip spot surface were lased 10 times for ionization of the proteins.

\section{Peak detection of SELDI protein profiles}

All the obtained spectra were internally mass-calibrated and normalized to the total ion current of an $\mathrm{m} / \mathrm{z}$ value more than 1000 for avoiding the signal interference from EAM. Expression difference mapping (EDM) was performed automatically using Ciphergen Express data management software version 3.0.6 under the following conditions: signal/noise ratio of 5 or higher for the first pass, 2 for the second pass, and presentation in at least $20 \%$ of spectra for identification.

\section{Data analysis for single biomarker candidate protein identification}

A comparative analysis of protein profiles of the MMD and control groups using univariate analysis was performed for single biomarker candidate protein identification under each $\mathrm{pH}$ condition. $\mathrm{P}$ values were calculated based on the Mann-Whitney $U$ test for nonparametric data or two-tailed $t$-test for parametric data, and the area under the receiver operation characteristic (ROC) curve (AUC) of each peak cluster was determined by Ciphergen Express data management software version 3.0. A P value of less than 0.05 was considered statistically significant.

\section{Data mining using classification and regression trees (CART)}

Generally, stability and accuracy of the prediction model were tested to assign a training set and test set from the data set. However, as the size of the data set in this study was too small to construct an independent validation set, a tree-based model was adopted to make a prediction model for multi-biomarker candidate protein identification. This model applies non-linear regression analysis and has recently been used broadly for data mining. CART for data mining was applied as described previously [26,27]. A decision tree was generated using entropy and the Gini index for calculating a node [28]. Branching of the tree is created based on the values (node) calculated by these indices and pruned using the complexity parameter. In this study, the complexity parameter was set to 0.01 . This analysis was performed with the $\mathrm{R}$ software environment for statistical computing ( $\mathrm{R}$ Development Core Team, Vienna, Austria). 


\section{Peak reproducibility}

Reproducibility of peak intensity and mass accuracy were evaluated using pooled CSF samples from two individuals from the control group. The coefficient of variance (CV) was calculated using randomly selected multiple protein peaks over the experiment as previously described $[29,30]$. For the assessment of peak reproducibility of the SELDI profiles, both the intra-assay (spot-to-spot) and inter-assay (chip-to-chip) CV were determined.

\section{Results}

\section{Peak reproducibility}

In this study, the intra-assay (spot-to-spot) CV was $17.38 \%$ for peak intensity and $0.11 \%$ for mass accuracy. The inter-assay (chip-to-chip) CV was $25.11 \%$ for peak intensity and $0.14 \%$ for mass accuracy, indicating acceptable reproducibility of the spectra.

\section{SELDI-TOS-MS protein profiling and data analysis SELDI-TOF-MS analysis for CSF samples}

After making the EDM cluster, the CSF samples were analyzed in a binding/washing buffer with three different $\mathrm{pH}$ conditions ( $\mathrm{pH} \mathrm{5,7}$ and 9). Between 1 to $100 \mathrm{kDa}$ $\mathrm{m} / \mathrm{z}, 54,40$ and $59 \mathrm{~m} / \mathrm{z}$ peak clusters were generated for the $\mathrm{pH} 5,7$ and 9 conditions, respectively; the respective minimum peaks were 1027.73, 1041.82 and 1026.39, and the respective maximum peaks were $93797.02,94648.40$ and 95278.35 .

\section{Univariate analysis using EDM data for identification of} single biomarker candidate proteins

For identification of single biomarker candidates in each $\mathrm{pH}$ condition, univariate analysis was performed using EDM data. Table 2 shows 34 biomarker candidate proteins differentially expressed among the MMD and control groups $(\mathrm{P}<0.05) ; \mathrm{m} / \mathrm{z}$ was less than $10 \mathrm{kDa}$ and ranged from 1041.03 to 50989.55 , for which 15 proteins were significant $(\mathrm{P}<0.01)$. Of the 34 biomarker candidates, 15, 5 and 4 were detected under $\mathrm{pH} 5,7$ and 9 conditions, respectively. The mean ROC AUC value of each cluster was 0.7723 , ranging from 0.7 to 0.9 . Among these protein candidates, 19 were up-regulated and 15 were down-regulated in the MMD group compared to the control group. There were 11 peak clusters thought to be originated from $5 \mathrm{~m} / \mathrm{z}$ peak clusters; m/z 4473.46 at $\mathrm{pH} 5$ and 4475.10 at $\mathrm{pH} 7, \mathrm{~m} / \mathrm{z} 4589.34$ at $\mathrm{pH} 9$, 4588.73 at $\mathrm{pH} 5$ and 4588.87 at $\mathrm{pH} 7, \mathrm{~m} / \mathrm{z} 6941.48$ at $\mathrm{pH} 9$ and 6942.96 at $\mathrm{pH} 7, \mathrm{~m} / \mathrm{z} 13882.19$ at $\mathrm{pH} 9$ and 13877.49 at $\mathrm{pH}$ 7. Within these clusters, there is a minimal difference in $\mathrm{m} / \mathrm{z}(<5.3)$. Spectra of representative single biomarker candidates under each $\mathrm{pH}$ condition using SELDI ProteinChip analysis are shown in Figure 1 for each CSF sample from the MMD and control groups. All proteins were up-regulated in the MMD
Table 2 Single biomarker candidates from univariate analysis of CSF sample comparison of MMD and control groups

\begin{tabular}{|c|c|c|c|c|}
\hline$P$-value* & $\mathrm{m} / \mathrm{z}$ & $\begin{array}{l}\text { pH of binding/ } \\
\text { washing buffer }\end{array}$ & $\begin{array}{l}\text { ROC } \\
\text { AUC }\end{array}$ & $\begin{array}{l}\text { Level in } \\
\text { MMD }\end{array}$ \\
\hline 1.60E-04 & 4746.76 & pH 9 & 0.9000 & up \\
\hline 5.32E-04 & 4566.71 & $\mathrm{pH} 5$ & 0.8583 & down \\
\hline $6.14 \mathrm{E}-04$ & $4473.46^{a}$ & pH 5 & 0.8750 & up \\
\hline $1.08 \mathrm{E}-03$ & 4157.65 & $\mathrm{pH} 9$ & 0.8250 & up \\
\hline 1.41E-03 & $4589.34^{b}$ & pH 9 & 0.8250 & up \\
\hline 1.41E-03 & 50989.55 & pH 9 & 0.8417 & down \\
\hline 1.41E-03 & $4588.73^{b}$ & pH 5 & 0.8250 & up \\
\hline 3.09E-03 & $4588.87^{b}$ & $\mathrm{pH} 7$ & 0.8000 & up \\
\hline 3.09E-03 & $6941.48^{d}$ & $\mathrm{pH} 9$ & 0.8083 & down \\
\hline $3.51 \mathrm{E}-03$ & $6942.96^{d}$ & $\mathrm{pH} 7$ & 0.8167 & down \\
\hline 7.24E-03 & 9656.51 & pH 5 & 0.8000 & up \\
\hline 7.24E-03 & 13754.72 & pH 5 & 0.7750 & up \\
\hline $8.12 \mathrm{E}-03$ & $4809.94^{c}$ & $\mathrm{pH} 5$ & 0.7667 & down \\
\hline $9.11 \mathrm{E}-03$ & 2406.55 & $\mathrm{pH} 5$ & 0.7667 & down \\
\hline $9.11 \mathrm{E}-03$ & $13882.19^{e}$ & pH 9 & 0.7583 & down \\
\hline $1.02 \mathrm{E}-02$ & 9728.65 & pH 5 & 0.7750 & up \\
\hline 1.14E-02 & 6228.21 & $\mathrm{pH} 5$ & 0.7333 & down \\
\hline 1.27E-02 & $13877.49^{e}$ & $\mathrm{pH} 7$ & 0.7917 & down \\
\hline $1.42 \mathrm{E}-02$ & 21299.65 & pH 5 & 0.7583 & down \\
\hline $1.58 \mathrm{E}-02$ & 5781.11 & $\mathrm{pH} 5$ & 0.7500 & up \\
\hline 1.76E-02 & 14060.91 & pH 9 & 0.7417 & down \\
\hline 1.76E-02 & 4695.40 & $\mathrm{pH} 5$ & 0.7417 & down \\
\hline 1.95E-02 & 6240.88 & pH 9 & 0.7500 & up \\
\hline 2.16E-02 & 28095.59 & pH 9 & 0.7583 & down \\
\hline 2.40E-02 & $4475.1^{a}$ & pH 7 & 0.7500 & up \\
\hline 2.40E-02 & 7250.89 & pH 7 & 0.7250 & up \\
\hline 2.40E-02 & 3516.49 & pH 9 & 0.7250 & up \\
\hline 2.93E-02 & 6888.58 & $\mathrm{pH} 5$ & 0.7250 & up \\
\hline $3.23 \mathrm{E}-02$ & 3681.83 & pH 9 & 0.7250 & up \\
\hline 3.56E-02 & 1041.03 & pH 9 & 0.7000 & up \\
\hline $3.91 \mathrm{E}-02$ & 7146.84 & $\mathrm{pH} 5$ & 0.7333 & down \\
\hline 4.30E-02 & $4811.33^{c}$ & pH 9 & 0.7000 & up \\
\hline 4.71E-02 & 1061.94 & pH 9 & 0.7250 & up \\
\hline 4.71E-02 & 34344.75 & pH 5 & 0.7083 & down \\
\hline
\end{tabular}

${ }^{\mathrm{a}-\mathrm{e}}$ Values with the same lower-case letter indicate clusters most likely derived from the same proteins. ${ }^{*} P<0.05$, Mann-Whitney $U$ test

group. A box-whisker plot of the peak intensities for representative single biomarker candidate proteins in each group is demonstrated in Figure 2. For the MMD group, the mean peak intensity of $\mathrm{m} / \mathrm{z} 4473$ at $\mathrm{pH} 5$ was $22.64 \pm 9.72$ (mean \pm standard deviation), that of $\mathrm{m} / \mathrm{z} 4588$ at $\mathrm{pH} 7$ was $56.67 \pm 28.42$, and that of $\mathrm{m} / \mathrm{z}$ 4746 at $\mathrm{pH} 9$ was $44.94 \pm 18.76$; those of the control group were $11.78 \pm 5.1630 .62 \pm 8.42$, and $20.37 \pm$ 10.24 , respectively. All peak intensities of each cluster were significantly larger in the MMD than control group $(\mathrm{P}<0.01)$. 


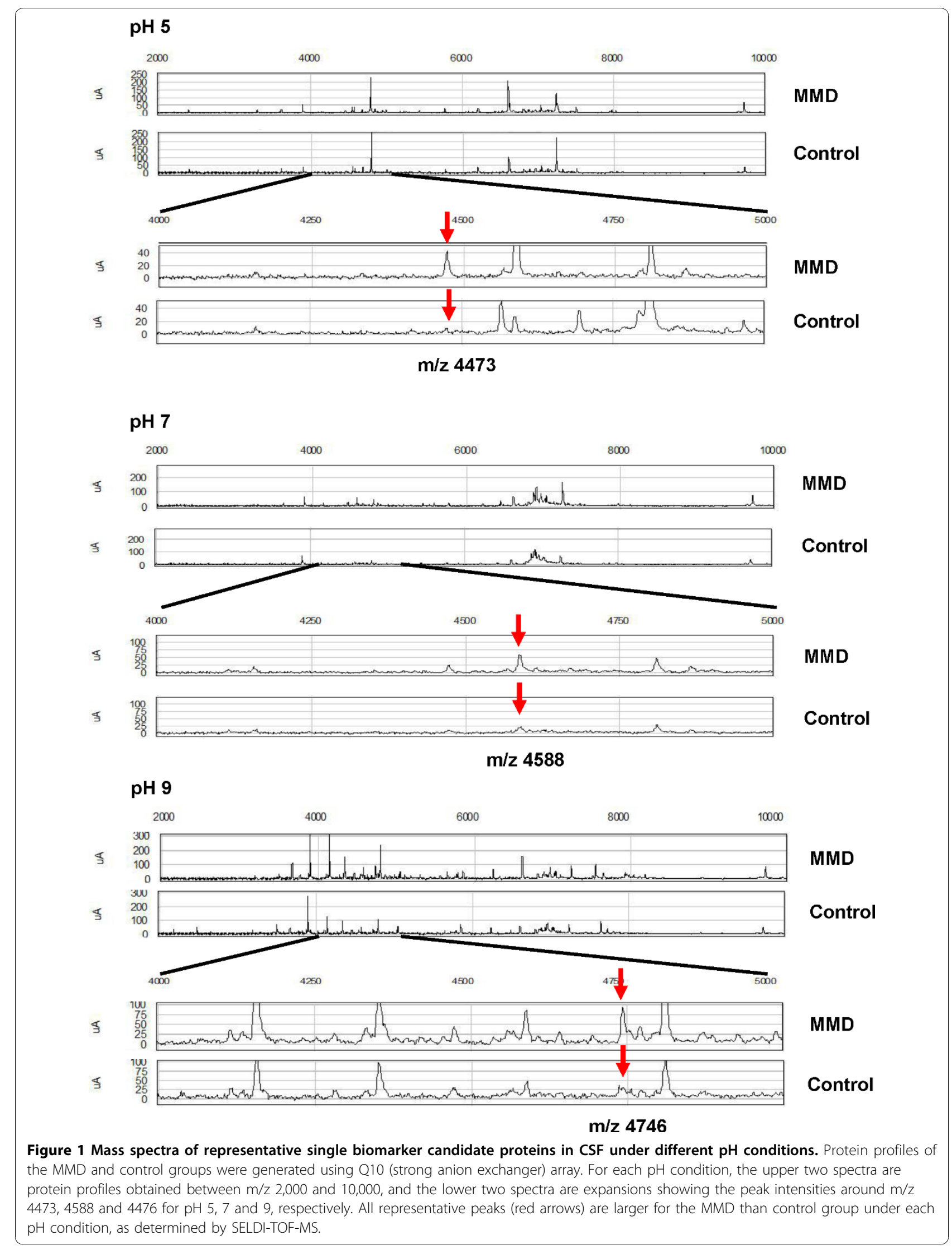



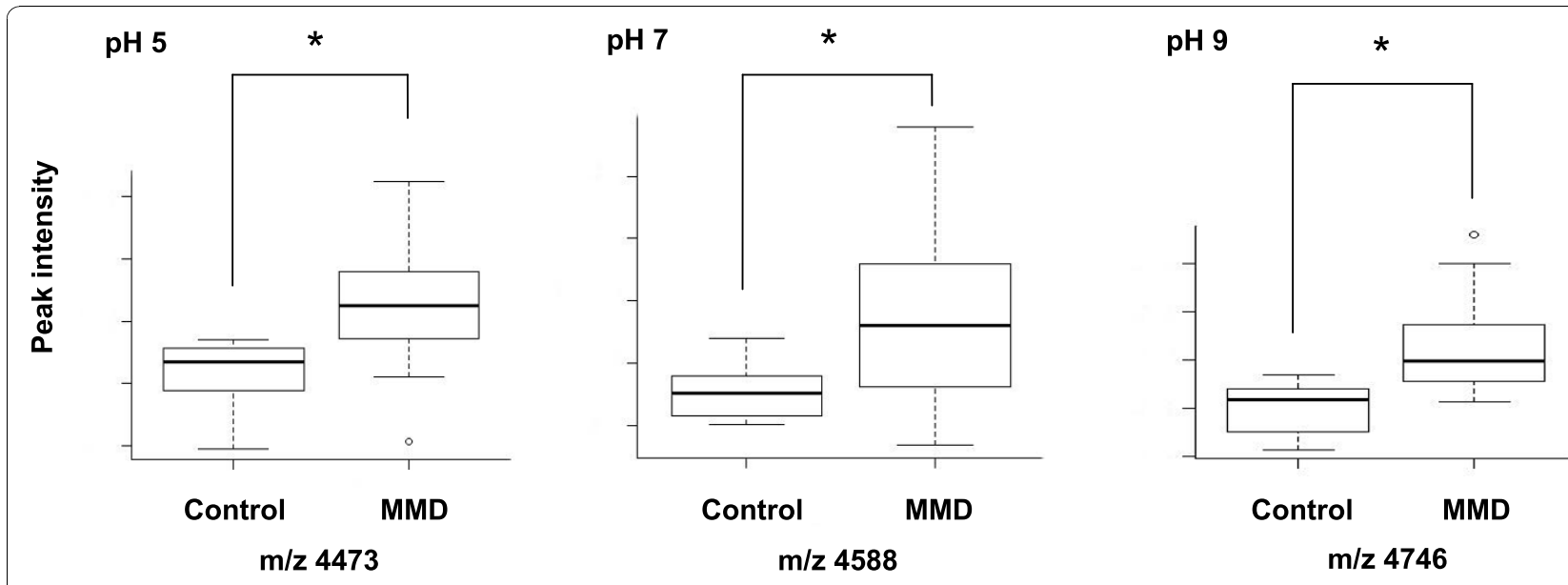

Figure 2 Peak intensity of representative single biomarker candidate proteins in the MMD and control groups. Peak intensities of representative single biomarker candidate proteins detected using SELDI-TOF-MS are significantly larger in the MMD than control group under each pH condition ( $\mathrm{m} / \mathrm{z} 4473,4588$ and 4476 for pH 5, 7 and 9, respectively). The box-whisker plots indicate the median value (thick line) and the 25th (lower line of box) and 75th (upper line of box) percentile; $T$ bars indicate the 10th and 90th percentile. The $p$ values between the groups were calculated using Mann-Whitney $U$ test. ${ }^{*} P<0.01$.

\section{Data mining using CART analysis}

CART analysis was undergone to discriminate patients with MMD from control patients using a single biomarker obtained by SELDI-TOF-MS under different $\mathrm{pH}$ conditions ( $\mathrm{pH} \mathrm{5,7}$ and 9) (Figure 3). The analysis correctly classified all 20 patients with MMD at $\mathrm{pH} 5$ and 19 of them at pH 7 and 9; all control patients were classified correctly under all $\mathrm{pH}$ conditions. As shown in Figure 4, CART analysis was able to classify 19 of the 20 patients with MMD and all 12 of the control patients.

\section{Discussion}

\section{Purpose of this study}

Diagnosis of MMD is currently only achievable by radiological features using cerebral angiography or magnetic resonance imaging/angiography. However, characteristics of MMD-mimicking vascular conditions are frequently encountered on radiological imaging in the clinical setting $[31,32]$, sometimes rendering indeterminate diagnosis of MMD. In this pilot study, we utilized novel biomarker candidate proteins identified in CSF samples by SELDI-TOF-MS to aid in the definite diagnosis of MMD. Based on our results, a mechanism for these biomarkers in association with the pathogenesis of MMD could be elucidated.

\section{Study design}

Many factors have limited the advancement of basic research on MMD. Some of these include low mortality rate, difficulty of obtaining surgical specimens from the internal carotid artery or related intracranial vessels, and lack of an appropriate animal model [33]. Recent investigations of MMD have focused on its epidemiology [2-4], pathology $[9,10]$, relationship with endothelial progenitor cells [16,17] and genetics [5-8], identifying some potential mechanisms and development pathways of this condition. However, their findings constitute only part of the underlying cause of MMD. As the CSF contacts the extracellular space of the brain, it contains an obvious source of biomarkers which should reflect central neuro-pathologic impairments of the brain. With the development of global proteomic research for biomarker discovery [34], previous studies have proposed many biomarkers, especially for neuro-degenerative diseases, although mainly using two-dimensional polyacrylamide electrophoresis $[35,36]$. A variety of recent proteomic techniques based on MS have also been utilized, including electrospray ionization tandem MS, matrix-assisted laser desorption/ionization MS and SELDI MS, with mechanically different methods of ion separation, mass accuracy and resolution, along with different pretreatments of low abundant proteins, such as found in the CSF $[37,38]$. Our study focused on proteomic analysis using SELDI-TOF-MS, a high-throughput, reliable technique which allows large numbers of patient samples to be investigated with a low sample volume scale, simple maneuvering and comparatively shorter time than other methods. SELDI technology has been used for a number of clinical situations and sample types to identify biomarker protein expression patterns which discriminate patients with a certain disease from control individuals [39].

We tested Q10 (strong anion exchanger) ProteinChip array to capture a wide variety of proteins derived from the CSF, including physiologically active substances 


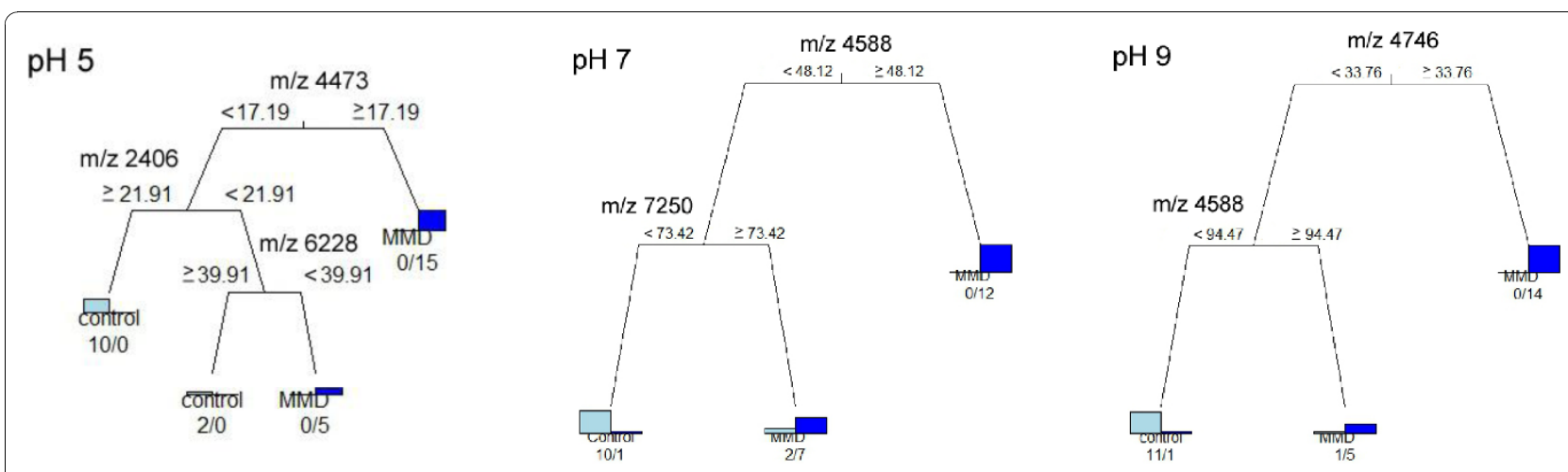

Figure 3 CART analysis using peaks obtained by SELDI-TOF-MS to discriminate between patients with MMD and control patients. The decision tree was constructed using CSF samples from 32 patients with MMD and control patients. The classification is determined starting at the roof node, following by appropriate splitting decisions based on the peak intensity at each node. If the peak intensity is lower than the cutoff intensity value, the left node is selected. This splitting process is continued until no further classification is achieved and terminal nodes are produced. Using m/z 4473, 2406 and 6338 peaks ( $\mathrm{pH}$ 5), m/z 4588 and 7250 peaks (pH 7), and m/z 4746 and 1044 peaks (pH 9), CART for Q10 ProteinChip was applied to identify patients with MMD and control patients. The analysis correctly classified all 20 patients with MMD under $\mathrm{pH} 5$ condition and 19 of 20 under the pH 7 and 9 conditions; all 12 control patients were classified under all pH conditions.

ionized under physiological $\mathrm{pH}(7.2$ - 7.4). We also tested CM10 (weak cation exchanger) ProteinChip array, which can indiscriminately detect many kinds of biomarker proteins, although no meaningful results were obtained using our methodology (data not shown). Q10 ProteinChip array was therefore applied in this study using a binding/washing buffer at $\mathrm{pH} 5,7$, and 9,

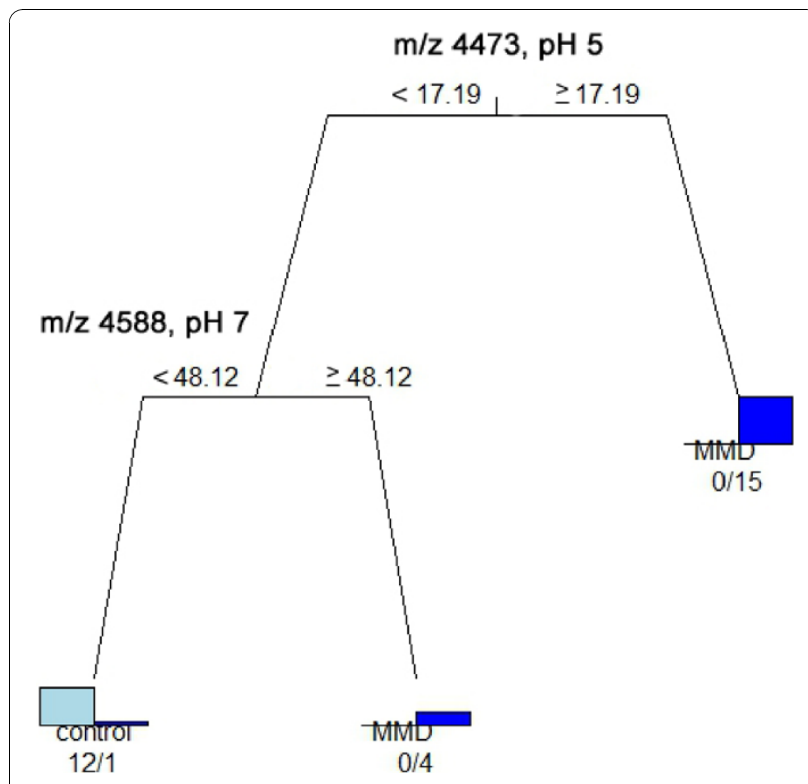

Figure 4 CART analyses using all 34 single biomarker candidate proteins. CART was analyzed for 34 single biomarker candidate proteins identified in the CSF under each pH condition ( $\mathrm{pH} \mathrm{5,7}$ and 9) to discriminate patients with MMD from control patients. This analysis correctly classified 19 of 20 patients with MMD and all 12 control patients based on the peak intensities of the $\mathrm{m} / \mathrm{z} 4473$ peak ( $\mathrm{pH}$ 5) and m/z 4588 peak (pH 7). around physiological $\mathrm{pH}$ conditions. This is the first study to identify biomarker candidate proteins by SELDI-TOF-MS using the CSF of patients with MMD.

\section{Evaluation of the results in this study}

Definite diagnostic tool for discrimination of patients with MMD and control patients

In the course of our study using SELDI-TOF-MS, 34 candidates of single biomarker proteins within the range of 1-50 kDa were generated with Q10 ProteinChip array. SELDI technology can feasibly resolve these low molecular weight proteins which cannot be resolved by other current proteomic methods like two-dimensional electrophoresis. As a definite diagnostic tool of MMD, analysis of these proteins could adequately discriminate between the MMD and control groups $(P<0.05$ and ROC AUC 0.7 - 0.9). CART analysis enabled this discrimination to provide a prediction model with relatively higher sensitivity and specificity, although the study population was too small to construct independent training and test set subjects. Therefore, a larger study population is needed for validation of these results. Comparison analysis using over-expressed/down-regulated proteins in the CSF of patients with MMD

Previous studies have suggested several growth factors that are related to angiogenesis. These include transforming growth factor- $\beta$ [11], basic fibroblast growth factor [12], hepatocyte growth factor [13], hypoxia inducible factor-1 [14] and vascular endothelial growth factor [15], with $\mathrm{m} / \mathrm{z}$ 83133, 55960, 42005, 92670, $38200 \mathrm{Da}$, respectively. As these angiogenic factors have relatively larger molecular weights $(>10 \mathrm{kDa})$, they are more accessible to purification and exact identification using conventional methodologies. For a further understanding of the 
Table 3 Biomarker candidate proteins (peptides) in the CSF of patients with MMD

\begin{tabular}{cl}
\hline Theoretical MW $(\mathbf{D a})$ & Inferred candidate protein (peptide) \\
\hline 4449.89 & Oxyntomodulin (P01275) \\
4451.26 & Urocortin-2 (Q96RP3) \\
4461.22 & Beta-defensin 133 (Q30KQ1) \\
4493.32 & Antibacterial protein LL-37 (P49913) \\
4585.34 & Liver-expressed antimicrobial peptide 2 (Q969E1) \\
4586.6 & Proenkephalin-A (143-183) (P01210)
\end{tabular}

The number in parentheses is the serial number of the protein in the UniProt Knowledgebase (Swiss-Prot and TrEMBL) databases.

underlying pathophysiology of MMD, the most significant $\mathrm{m} / \mathrm{z}$ peaks were elucidated on the proteome-wide database level. Candidate protein (peptide) biomarkers were inferred using the TagIdent tool (http://au.expasy. org/tools/tagident.html) from the UniProt Knowledgebase (Swiss-Prot and TrEMBL) databases based on the definite molecular mass; the retrieval conditions were set as $\mathrm{pI} 1.0$ to 14.0 and $\mathrm{m} / \mathrm{z} \pm 0.5 \%$. Six proteins (peptides) had corresponding molecular weights of two candidates, $\mathrm{m} / \mathrm{z} 4473$ and 4588 , obtained by CART analysis from the 34 single biomarker candidate proteins (Table 3). Exact identification of biomarker proteins is undoubtedly advantageous, although as in this study, purification is difficult to achieve with a lack of abundant samples from clinical subjects. Moreover, protein identification requires specialized techniques, and a highly sensitive method is needed to properly identify the target protein, especially those with a low molecular weight.

\section{Conclusions}

Several novel biomarker candidate proteins differentially expressed in patients with MMD and control patients were identified using a recently developed proteomic approach. This is a pilot study for CSF proteomics of MMD using SELDI technology. Multi-biomarker analysis using CART made the prediction models more specific and exhibited two $\mathrm{m} / \mathrm{z}$ panels as a discriminative tool for the definite diagnosis of MMD. For further understanding of the pathogenesis of MMD, a larger number of CSF samples for validation of the analysis and welldefined methodology and techniques for exact identification of biomarkers are essential.

\section{Acknowledgements \\ We are grateful to Mr. Minoru Fukayama (Aichi Medical University) for his technical assistance with SELDI data acquisition.}

\section{Author details}

${ }^{1}$ Department of Neurosurgery, Nagoya University Graduate School of Medicine, 65 Tsurumai-cho, Showa-ku, Nagoya, Aichi 466-8550, Japan. ${ }^{2}$ Promoting Center for Clinical Research, Aichi Medical University School of Medicine, Nagakute-cho, Aichi 480-1195, Japan. ${ }^{3}$ Center for Genetic and Regenerative Medicine, Nagoya University Hospital, 65 Tsurumai-cho, Showaku, Nagoya, Aichi 466-8550, Japan.

\section{Authors' contributions}

YA and KY carried out SELDI and participated in the subject evaluations. YA, SO, SM and MM contributed to collecting CSF samples. YA, KY, SO and TW participated in study design and coordination and drafting the manuscript. All authors read and approved the final manuscript.

\section{Competing interests}

The authors declare that they have no competing interests.

Received: 31 May 2010 Accepted: 8 November 2010

Published: 8 November 2010

\section{References}

1. Suzuki J, Takaku A: Cerebrovascular "moyamoya" disease. Disease showing abnormal net-like vessels in base of brain. Arch Neurol 1969, 20:288-299.

2. Wakai K, Tamakoshi A, Ikezaki K, Fukui M, Kawamura T, Aoki R, Kojima M, Lin Y, Ohno Y: Epidemiological features of moyamoya disease in Japan: findings from a nationwide survey. Clin Neurol Neurosurg 1997, 99(Suppl): S1-S5.

3. Goto $Y$, Yonekawa Y: Worldwide distribution of moyamoya disease. Neurol Med Chir (Tokyo) 1992, 32:883-886.

4. Mineharu Y, Takenaka K, Yamakawa H, Inoue K, Ikeda H, Kikuta KI, Takagi Y, Nozaki K, Hashimoto N, Koizumi A: Inheritance pattern of familial moyamoya disease: autosomal dominant mode and genomic imprinting. J Neurol Neurosurg Psychiatry 2006, 77:1025-1029.

5. Ikeda H, Sasaki T, Yoshimoto T, Fukui M, Arinami T: Mapping of a familial moyamoya disease gene to chromosome 3p24.2-p26. Am J Hum Genet 1999, 64:533-537.

6. Sakurai K, Horiuchi Y, Ikeda H, Ikezaki K, Yoshimoto T, Fukui M, Arinami T: A novel susceptibility locus for moyamoya disease on chromosome 8q23. J Hum Genet 2004, 49:278-281.

7. Inoue TK, Ikezaki K, Sasazuki T, Matsushima T, Fukui M: Linkage analysis of moyamoya disease on chromosome 6. J Child Neurol 2000, 15:179-182.

8. Yamauchi T, Tada M, Houkin K, Tanaka T, Nakamura Y, Kuroda S, Abe H, Inoue T, Ikezaki K, Matsushima T, Fukui M: Linkage of familial moyamoya disease (spontaneous occlusion of the circle of Willis) to chromosome 17q25. Stroke 2000, 31:930-935.

9. Masuda J, Ogata J, Yutani C: Smooth muscle cell proliferation and localization of macrophages and T cells in the occlusive intracranial major arteries in moyamoya disease. Stroke 1993, 24:1960-1967.

10. Yamashita M, Oka K, Tanaka K: Histopathology of the brain vascular network in moyamoya disease. Stroke 1983, 14:50-58.

11. Hojo M, Hoshimaru M, Miyamoto S, Taki W, Nagata I, Asahi M, Matsuura N,

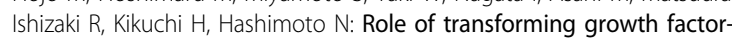
beta1 in the pathogenesis of moyamoya disease. J Neurosurg 1998, 89:623-629.

12. Malek AM, Connors S, Robertson RL, Folkman J, Scott RM: Elevation of cerebrospinal fluid levels of basic fibroblast growth factor in moyamoya and central nervous system disorders. Pediatr Neurosurg 1997, 27:182-189.

13. Nanba R, Kuroda S, Ishikawa T, Houkin K, Iwasaki Y: Increased expression of hepatocyte growth factor in cerebrospinal fluid and intracranial artery in moyamoya disease. Stroke 2004, 35:2837-2842.

14. Takagi Y, Kikuta K, Nozaki K, Fujimoto M, Hayashi J, Imamura H, Hashimoto N: Expression of hypoxia-inducing factor-1 alpha and endoglin in intimal hyperplasia of the middle cerebral artery of patients with Moyamoya disease. Neurosurgery 2007, 60:338-345.

15. Yoshimoto $\mathrm{T}$, Houkin $\mathrm{K}$, Takahashi A, Abe H: Angiogenic factors in moyamoya disease. Stroke 1996, 27:2160-2165.

16. Asahara T, Murohara T, Sullivan A, Silver $M$, van der Zee $R$, Li T, Witzenbichler B, Schatteman G, Isner JM: Isolation of putative progenitor endothelial cells for angiogenesis. Science 1997, 275:964-967.

17. Ceradini DJ, Gurtner GC: Homing to hypoxia: HIF-1 as a mediator of progenitor cell recruitment to injured tissue. Trends Cardiovasc Med 2005, 15:57-63.

18. Fuse T, Takagi T, Fukushima T, Hashimoto N, Yamada K: Arteriovenous malformation associated with moyamoya disease. Childs Nerv Syst 1996, 12:404-408.

19. Cho WC: Contribution of oncoproteomics to cancer biomarker discovery. Mol Cancer 2007, 6:25 
20. Cho WC, Cheng CH: Oncoproteomics: current trends and future perspectives. Expert Rev Proteomics 2007, 4:401-410.

21. Stoop MP, Coulier L, Rosenling T, Shi S, Smolinska AM, Buydens L, Ampt K, Stingl C, Dane A, Muilwijk B, Luitwieler RL, Smitt PA, Hintzen RQ, Bischoff R, Wijmenga SS, Hankemeier T, van Gool AJ, Luider TM: Quantitative proteomics and metabolomics analysis of normal human cerebrospinal fluid samples. Mol Cell Proteomics 2010, 9:2063-2075.

22. Issaq HJ, Veenstra TD, Conrads TP, Felschow D: The SELDI-TOF MS approach to proteomics: protein profiling and biomarker identification. Biochem Biophys Res Commun 2002, 292:587-592.

23. Simonsen AH, McGuire J, Podust VN, Davies H, Minthon L, Skoog I, Andreasen N, Wallin A, Waldemar G, Blennow K: Identification of a novel panel of cerebrospinal fluid biomarkers for Alzheimer's disease. Neurobiol Aging 2008, 29:961-968.

24. Ranganathan S, Williams E, Ganchev P, Gopalakrishnan V, Lacomis D, Urbinelli L, Newhall K, Cudkowicz ME, Brown RH Jr, Bowser R: Proteomic profiling of cerebrospinal fluid identifies biomarkers for amyotrophic lateral sclerosis. J Neurochem 2005, 95:1461-1471.

25. Research on intractable diseases of the Ministry of Health, Labour and Welfare, Japan: Recommendations for the Management of Moyamoya Disease: A Statement from Research Committee on Spontaneous Occlusion of the Circle of Willis (Moyamoya Disease). Surg Cereb Stroke 2009, 37:321-337, (in Japanese).

26. Kojima T, Yoshikawa K, Saga S, Yamada T, Kure S, Matsui T, Uemura T, Fujimitsu Y, Sakakibara M, Kodera Y, Kojima H: Detection of elevated proteins in peritoneal dissemination of gastric cancer by analyzing mass spectra data of serum proteins. J Surg Res 2009, 155:13-17.

27. Su Y, Shen J, Qian H, Ma H, Ji J, Ma H, Ma L, Zhang W, Meng L, Li Z, Wu J, Jin G, Zhang J, Shou C: Diagnosis of gastric cancer using decision tree classification of mass spectral data. Cancer Sci 2007, 98:37-43.

28. Gianola D, Perez-Enciso M, Toro MA: On marker-assisted prediction of genetic value: beyond the ridge. Genetics 2003, 163:347-365.

29. Kanmura S, Uto H, Kusumoto K, Ishida Y, Hasuike S, Nagata K, Hayashi K, Ido A, Stuver SO, Tsubouchi H: Early diagnostic potential for hepatocellular carcinoma using the SELDI ProteinChip system. Hepatology 2007, 45:948-956.

30. Scarlett CJ, Saxby AJ, Nielsen A, Bell C, Samra JS, Hugh T, Baxter RC, Smith RC: Proteomic profiling of cholangiocarcinoma: diagnostic potential of SELDI-TOF MS in malignant bile duct stricture. Hepatology 2006, 44:658-666.

31. Braun KP, Bulder MM, Chabrier S, Kirkham FJ, Uiterwaal CS, Tardieu M, Sebire G: The course and outcome of unilateral intracranial arteriopathy in 79 children with ischaemic stroke. Brain 2009, 132:544-557.

32. Sebire G: Transient cerebral arteriopathy in childhood. Lancet 2006, 368:8-10.

33. Kim SK, Yoo Jl, Cho BK, Hong SJ, Kim YK, Moon JA, Kim JH, Chung YN, Wang KC: Elevation of CRABP-I in the cerebrospinal fluid of patients with Moyamoya disease. Stroke 2003, 34:2835-2841.

34. Cho WC: Proteomics technologies and challenges. Genomics Proteomics Bioinformatics 2007, 5:77-85.

35. Rohlff C, Southan C: Proteomic approaches to central nervous system disorders. Curr Opin Mol Ther 2002, 4:251-258.

36. Zheng PP, Luider TM, Pieters R, Avezaat CJ, van den Bent MJ, Sillevis Smitt PA, Kros JM: Identification of tumor-related proteins by proteomic analysis of cerebrospinal fluid from patients with primary brain tumors. $J$ Neuropathol Exp Neurol 2003, 62:855-862.

37. Pan S, Zhu D, Quinn JF, Peskind ER, Montine TJ, Lin B, Goodlett DR, Taylor G, Eng J, Zhang J: A combined dataset of human cerebrospinal fluid proteins identified by multi-dimensional chromatography and tandem mass spectrometry. Proteomics 2007, 7:469-473.

38. Romeo MJ, Espina V, Lowenthal M, Espina BH, Petricoin EF, Liotta LA: CSF proteome: a protein repository for potential biomarker identification. Expert Rev Proteomics 2005, 2:57-70.

39. Cho WC: Research progress in SELDI-TOF MS and its clinical applications. Sheng Wu Gong Cheng Xue Bao 2006, 22:871-876.

\section{Pre-publication history}

The pre-publication history for this paper can be accessed here:

http://www.biomedcentral.com/1471-2377/10/112/prepub

\section{doi:10.1186/1471-2377-10-112}

Cite this article as: Araki et al:: Identification of novel biomarker candidates by proteomic analysis of cerebrospinal fluid from patients with moyamoya disease using SELDI-TOF-MS. BMC Neurology 2010 10:112.

\section{Submit your next manuscript to BioMed Central and take full advantage of:}

- Convenient online submission

- Thorough peer review

- No space constraints or color figure charges

- Immediate publication on acceptance

- Inclusion in PubMed, CAS, Scopus and Google Scholar

- Research which is freely available for redistribution

Submit your manuscript at www.biomedcentral.com/submit
Ciomed Central 